ARTIGOS

Submetido em 26.06.2013. Aprovado em 03.02.2014

Avaliado pelo sistema double blind review. Editora Científica: Sonia Maria Fleury Teixeira

DOI: http://dx.doi.org/10.1590/So034-759020140608

\title{
ORGANIZAÇÃO E DESEMPENHO: AVALIAÇÃO DA CENTRALIZAÇÃO DA PATOLOGIA DO INCA-BRASIL
}

\author{
Organization and performance: assessing centralization of pathology at \\ INCA-Brazil \\ Organización y desempeño: evaluación de la centralización de la patología del \\ INCA-Brasil
}

\begin{abstract}
RESUMO
A literatura atribui à descentralização da estrutura organizacional efeitos positivos sobre a orientação empreendedora e o desempenho da firma; existem também evidências de efeito positivo da centralização dos procedimentos complexos em hospitais de grande porte sobre os resultados do tratamento do câncer. $\mathrm{O}$ objetivo deste estudo é avaliar os efeitos da centralização dos laboratórios de anatomia patológica do Instituto Nacional do Câncer (INCA) brasileiro, em 2002, sobre o desempenho da atividade de diagnóstico e controle do câncer. A análise é desenvolvida com o cálculo de uma fronteira eficiente não paramétrica de produção no período 1997-2007, por meio da Análise Envoltória de Dados (DEA). 0 resultado é que a centralização reverteu a queda de eficiência técnica dos laboratórios no período 1997-2001. A conclusão do artigo é que o Modelo DEA traz um aporte ao conhecimento sobre a mudança de estrutura organizacional nas organizações públicas de saúde e uma contribuição gerencial sobre a eficácia da centralização para melhorar o suporte laboratorial da patologia aos hospitais do INCA.
\end{abstract}

PALAVRAS-CHAVE | Estrutura organizacional, centralização, Hospital do Câncer - Brasil, anatomia patológica, Análise Envoltória de Dados.

\section{MARCELINO JOSÉ JORGE marcelino.jorge@ipec.fiocruz.br Coordenador do Laboratório de Pesquisa em Economia das Organizações de Saúde do Instituto Nacional de Infectologia Evandro Chagas, Fundação Oswaldo Cruz - Rio de Janeiro - RJ, Brasil}

\section{FREDERICO A. DE CARVALHO} decarvalhofred@hotmail.com Professor da Faculdade de Administração e Ciências Contábeis, Universidade Federal do Rio de Janeiro - Rio de Janeiro - RJ, Brasil

\section{RAFAEL PESSOA SANTIAGO rafaelpessoa_rj@yahoo.com.br Assistente Contábil da Gerência de Retaguarda, Caixa Econômica Federal - Rio de Janeiro - RJ, Brasil}

\section{DANIELA DE SOUZA FERREIRA} daniela.ferreira@ipec.fiocruz.br Suporte de Gestão do Laboratório de Pesquisa em Economia das Organizações de Saúde, Instituto Nacional de Infectologia Evandro Chagas, Fundação Oswaldo Cruz, Rio de Janeiro - RJ, Brasil

\begin{abstract}
Literature widely assigns positive effects on entrepreneurial orientation and firm performance to decentralization of organizational structure; at the same time, there is evidence of positive effects of centralization of complex procedures in large hospitals on the results of cancer treatment. This study intends to evaluate the effects of centralization of pathology laboratories at the National Cancer Institute (INCA) of Brazil in 2002 on the performance of diagnostic activity and cancer control. A non-parametric efficiency frontier for the pathology labs is calculated for the period 1997-2007 by means of Data Envelopment Analysis (DEA). Findings show that centralization reversed the decline of technical efficiency in the laboratories along the period 1997-2001. The main conclusion is that the DEA model brings a contribution to knowledge about change in organizational structure in public health organizations, as well as a managerial contribution on the effectiveness of centralization to improve the support of pathology labs to INCA hospitals.
\end{abstract}

KEYWORDS / Organizational structure, centralization, Brazilian National Cancer Institute, pathology laboratory, Data Envelopment Analysis.

\section{RESUMEN}

La literatura le atribuye a la descentralización de la estructura organizacional efectos positivos sobre la orientación emprendedora y el desempeño de la firma; también existen evidencias de efecto positivo de centralización de los procedimientos complejos en hospitales de gran porte sobre los resultados del tratamiento de cáncer. El objetivo de este estudio es evaluar los efectos de la centralización de los laboratorios de anatomía patológica del Instituto Nacional del Cáncer (INCA) brasileño, en 2002, sobre el desempeño de la actividad de diagnóstico y control del cáncer. El análisis es desarrollado con el cálculo de una frontera eficiente no paramétrica de producción en el periodo 1997-2007, por medio del Análisis Envolvente de Datos (DEA). El resultado es que la centralización revertió la caída de la eficiencia técnica de los laboratorios en el periodo 1997-2001. La conclusión del artículo es que el Modelo DEA presenta un aporte al conocimiento sobre el cambio de estructura organizacional en las organizaciones públicas de salud y una contribución gerencial sobre la eficacia de la centralización para mejorar el soporte del laboratorio de patología de los hospitales del INCA.

PALABRAS-CLAVE I Estructura organizacional, centralización, Hospital del Cáncer - Brasil, Anatomía Patológica, Análisis Envolvente de Datos. 


\section{INTRODUÇÃO}

A criação do Sistema Único de Saúde (SUS) tornou a universalidade da assistência um dos princípios fundamentais do sistema de saúde brasileiro, complementando os princípios da equidade e da integralidade dos serviços de saúde do Governo, incumbido da regulamentação, fiscalização e controle do sistema.

A reforma do Estado no Brasil, por sua vez, inspirou a gestão orientada para resultados em organizações públicas, tais como o Instituto Nacional de Câncer (INCA), nos anos 1990, e forjou o interesse por métodos de avaliação de performance (Faria, 2007).

Em particular, a literatura encoraja estudos sobre a operacionalização desses princípios com base na avaliação das diferentes estratégias individuais do âmbito das próprias organizações de saúde (Puccini, 2008).

0 estudo a respeito do efeito do volume de atividade dos hospitais - o número de casos tratados - sobre a efetividade dos esquemas de tratamento do paciente acometido de câncer, um tema controverso na literatura, é um exemplo de interesse para a pesquisa em Administração e Economia que contribui para o entendimento da estratégia de melhorar as condições efetivas de acesso ao diagnóstico do câncer pela população brasileira, por meio da centralização da gerência do Serviço de Anatomia Patológica (SAP) do INCA, promovida em 2002.

0 controle do câncer depende, de fato, de ações variadas, mas é a terapêutica especializada, de alto custo, que mais consome recursos. Em contrapartida, as chances de sucesso no tratamento são maiores com a detecção precoce de lesões precursoras ou câncer em fase inicial. O INCA dá suporte à formulação da política nacional de atenção em oncologia e promove ações nacionais integradas para prevenção e controle do câncer, envolvendo diagnóstico e tratamento especializado, reabilitação e cuidados paliativos. Além disso, realiza atividades de pesquisa e ensino (Faria, 2007).

Para esse escopo de atuação, o INCA está fragmentado em 18 unidades no Rio de Janeiro. Além do Hospital do Câncer ( $\left.\mathrm{HC}_{1}\right)$, conta com:

a. o HC2, incorporado ao INCA em 1992 e que é centro de referência em oncologia ginecológica;

b. o HC3, também incorporado em 1992, dedicado exclusivamente ao diagnóstico e ao tratamento do câncer de mama desde 1999; e

c. O HC4, a Unidade de Cuidados Paliativos do INCA, responsável pelo atendimento aos pacientes portadores de câncer avançado, sem possibilidade de cura.

Apenas os $\mathrm{HC}_{1}, \mathrm{HC}_{2}$ e HC 3 têm atividade de centro cirúrgico e requerem, portanto, um serviço de patologia, um Núcleo Hospi- talar de Patologia (NHP) com procedimentos padronizados, especialização de tarefas e elevado grau de treinamento do pessoal, a unidade operacional que concentrou a atenção deste estudo.

Do ponto de vista da sua estrutura organizacional global, o INCA adotou o regime de cogestão na década de 1980, quando a experiência internacional de centralização da cirurgia de câncer se notabilizou, e a unificação do SAP em 2002, respectivamente para promover internamente o ânimo empreendedor e solucionar problemas de coordenação e compromisso, e ocupou posição de pivô da política de controle do câncer no Brasil (Faria, 2007).

O SAP, em suma, é uma unidade produtiva especializada de uma organização pública multipropósito, com atividades de produção de laudos de diagnóstico, ensino e pesquisa, composta de três NHPs que têm linhas de produção idênticas e cuja gerência foi centralizada na Divisão de Patologia do INCA (Dipat) a partir do início da década passada (Santiago, 2009).

O desafio para avaliar organizações públicas com múltiplos objetivos tais como o INCA refere-se à dificuldade para estabelecer padrões de resultados sobre as unidades operacionais com estruturas organizacionais diferenciadas das organizações com unidades interdependentes (Vakkuri, 2003).

Diante da importância epidemiológica do câncer no Brasil, seu impacto social e os custos elevados do tratamento complexo, este estudo examina o efeito da centralização do SAP sobre o desempenho do INCA no uso de recursos.

\section{MARCO CONCEITUAL}

Esta seção examina os conceitos de estrutura organizacional, orientação empresarial e desempenho com que a literatura de pesquisa em Administração e Economia interna das organizações investiga as relações potenciais entre grau de centralização da tomada de decisão e vantagem competitiva (ou "sustentabilidade", para as organizações públicas). A seguir, apresenta a abordagem da literatura de Ciências da Saúde a respeito do efeito do volume de procedimentos de alta complexidade centralizados em hospital de grande porte sobre o controle do câncer e o resultado (functional outcome) alcançado com o tratamento cirúrgico.

\section{Estrutura organizacional, centralização, orientação empreendedora e desempenho}

Mintzberg (1995) definiu estrutura organizacional como o conjunto das formas de alocação e coordenação da força de trabaIho em suas tarefas. Caracterizou, entre elas, a burocracia profissional, a organização divisionalizada e a organização inovadora. 
Três dimensões da estrutura organizacional focalizadas na pesquisa sobre a origem da vantagem competitiva são a complexidade (i. e, a multiplicidade de divisões de função envolvidas), a formalização das normas e a centralização (i. e, a concentração, a unificação e a ampliação do alcance) da tomada de decisão (Morton \& Hu, 2008).

Nessa abordagem, o efeito positivo da centralização sobre o feedback obtido do quadro de especialistas é atribuído ao fato de a centralização tornar as diretrizes de política da organização mais uniformes e reduzir o risco de falta de informação do empregado (Katsikea, Theodosiou, Perdikis, \& Kehagias, 2011).

Sob esse prisma, a burocracia profissional adapta-se a um ambiente estável, com sistemas técnicos não regulados e não sofisticados. Emprega profissionais com alta padronização das habilidades, o trabalho é complexo e privilegia a contratação de profissionais treinados, detentores do controle sobre o seu próprio trabalho, com vistas à interiorização dos padrões. 0 mecanismo de coordenação típico é a padronização das habilidades.

Como esses padrões têm origem fora da estrutura, no caso de associações de profissionais autogovernadas, a burocracia profissional enfatiza a autoridade de natureza profissional e o poder do conhecimento, permitindo elevada autonomia aos profissionais. Nesse tipo de estrutura, é pequena a necessidade de supervisão direta sobre os profissionais ou de ajustamento mútuo entre eles e as unidades operacionais, de hierarquia, de formalização e de planejamento e controle.

Nessa estrutura, a administração detém o controle de considerável parte dos recursos e estabelece as diretrizes, os rumos e o projeto organizacional, fixando as prioridades para a alocação de recursos. Apesar disso, caracteriza-se por baixa governabilidade (Mintzberg, 1995).

$\mathrm{Na}$ organização divisionalizada, as unidades operacionais são diferenciadas pela padronização dos outputs, o que as torna suficientemente autônomas e com baixa hierarquia. A estrutura divisionalizada é adequada à organização constituída por unidades operacionais independentes, sob controle uniforme de um "escritório central"; cada divisão possui sua estrutura própria, diferente das demais, resultando em descentralização pronunciada. Em princípio, as unidades operacionais tomam as decisões concernentes às suas próprias operações, e o "escritório central" depois monitora os resultados dessas decisões (Mintzberg, 1995). Ao par de proporcionar autonomia, o “escritório central” deve manter alguma forma de controle sobre as unidades operacionais, sustentando a coordenação da organização como um todo: a padronização dos outputs também funciona como mecanismo de coordenação das unidades operacionais, mas o parâmetro-chave do desenho da organização é o sistema de controle de desempenho (Mintzberg, 1995).
Quando, em organizações públicas com múltiplos objetivos, tais como o INCA, a descentralização em estruturas divisionalizadas visa fortalecer a atividade de pesquisa orientada para resultados, surge, então, a questão sobre como o "escritório central" pode manter controle e permitir autonomia suficiente a cada divisão, se as unidades operacionais passam a ter autonomia restrita, pois seus outputs são escolhidos, a priori, por meio da definição do plano estratégico da organização, cujo monitoramento pelo "escritório central" é formalizado pela avaliação quantitativa do desempenho (Jorge, 2001).

A esse propósito, a atividade de pesquisa, sujeita a incertezas, é realizada em um ambiente dinâmico e, por ser de difícil avaliação, molda um ambiente complexo. 0 ambiente de pesquisa, portanto, também é compatível com o desenvolvimento da estrutura da organização inovadora, que exibe descentralização efetiva, focalizada na integração de profissionais de diferentes especialidades em equipes regulares de projetos ad hoc.

Associada à especialização das tarefas, porém sem padronização das habilidades, e devotada à adaptação rápida aos novos projetos, a organização inovadora é uma estrutura que exibe pouca formalização e centralização, planejamento limitado das ações, descentralização seletiva, dispersão da tomada de decisão, delegação do controle aos especialistas, dedicação de parcela importante do tempo do dirigente ao monitoramento dos projetos e ajuste mútuo como principal mecanismo de coordenação (Mintzberg, 1995). Também segundo Lumpkin, Cogliser e Schneider (2010), a centralização guarda estreita relação com a autonomia, a influência direta considerada de maior peso na orientação empreendedora do pessoal, à qual é atribuído importante efeito sobre o desempenho.

A literatura sobre vantagens competitivas assinala que as organizações se confrontam com situações que requerem atenção para atividades de orientação empreendedora e devem internalizar o espírito empreendedor para habilitar seu pessoal (Shepherd, Covin, \& Kuratko, 2008). Dessa necessidade, resulta a importância das organizações que encorajam o ânimo empreendedor e o potencial do indivíduo, assim como da estrutura organizacional que ofereça condições adequadas para a orientação empreendedora crescer (Johnson \& Ven, 2002). Destaca, ainda, que os métodos de tomada de decisão e as ações individuais são influenciados pelo conjunto das características da orientação empreendedora, tais como a atitude de competição agressiva, inovadora, proativa, de tomada de risco e de autonomia e que, combinadas, essas características melhoram o desempenho empreendedor da organização, que alcança suas metas com mais eficiência, desafia a concorrência e adquire capacidade adaptativa para enfrentar a mudança nos ambientes turbulentos (Jogaratnam \& Tse, 2006). 
Não obstante ter confirmado a estrutura organizacional como um dos fatores mais influentes sobre o estabelecimento das diferentes dimensões da orientação empreendedora, a investigação sobre o sucesso das organizações identificou uma relação inversa da autonomia e da variedade das ocupações com o grau de centralização da estrutura organizacional (Alimardani, Ghahremani, \& Abulghesemi, 2009).

A conclusão dessa literatura é que a estrutura da organização deve ser menos centralizada e envolver mais participação (Robinson, 2007). As soluções mais criativas e inovadoras surgem na organização que desfruta de estruturas descentralizadas e, para motivar novas ideias, a organização pública que adota a remuneração por desempenho deve levar em conta a estrutura organizacional e descentralizar (Caruana, Ewing, \& Ramaseshan, 2002).

A propósito da estrutura das organizações de saúde, a melhoria do desempenho em geral e de ambos os componentes da qualidade total - o design e a conformidade - requer simultaneamente um nível de formalização elevado e mais descentralização (Meirovich, Brender-llan, \& Meirovich, 2007).

Prevalece, em suma, a opinião de que a preocupação quanto à possibilidade da descentralização da governabilidade da atenção em saúde vir a resultar em mais desigualdade regional, privatização da atenção médica, ineficiências associadas à duplicação de atividades gerenciais e queda da produtividade decorre, em parte, da falta de avaliações mais rigorosas (Costa-I-Font, 2012).

\section{Tratamento do câncer: centralização, controle, treinamento e resultado}

Na literatura organizacional, Etzioni (1967, p. 49) foi um dos primeiros a destacar que a existência de unidades de serviço especializado influi na centralização porque tais unidades são demasiado especializadas e dispendiosas para ligar-se a níveis mais baixos na organização.

Em contrapartida, na literatura de Ciências da Saúde sobre o tratamento do câncer, o conceito "centralização" focaliza, para o conjunto do sistema de saúde - population-wide - a relação entre a quantidade de procedimentos cirúrgicos conduzidos em hospital de grande porte - high-volume centers - e as conseqüências para o paciente, ou seja, a vantagem de escala decorrente da mudança técnica - technology-driven (Harrison, 2012; Lemmens, Bosscha, Schelling, Brenninkmeijer, Coebergh, \& Hingh, 2011; Stitzenberg, Wong, Nielsen, Egleston, \& Uzzo, 2012).

Em termos quantitativos, a centralização é medida como a razão entre o volume de atividade do hospital, i.e, o número de casos tratados, e o resultado alcançado - outcome - definido como a variação com respeito à taxa de óbito hospitalar de 30 dias (Harrison, 2012).

Associada à noção de grandes unidades e cirurgiões realizando um número grande de operações ou de centros especializados versus centros com menos experiência, desde Luft, Bunker e Enthoven (1979), a concepção da centralização do tratamento de câncer nas Ciências da Saúde focaliza, em primeiro lugar, a importância da escala de produção e envolve a identificação e a adoção de processos já testados com sucesso em escalas elevadas e por sistemas de atenção de alta qualidade, além de estruturas de cooperação entre especialidades (Harrison, 2012; Lemmens, Bosscha, Schelling, Brenninkmeijer, Coebergh, \& Hingh, 2011).

Destaca, em segundo lugar, a importância do controle da informação: inobstante a atenção assumir diferentes formas e variar desde os planos de enfoque local até o do direcionamento dos pacientes de regiões geográficas amplas para centros de atenção terciária, a centralização facilita o monitoramento preciso dos padrões de toda a ampla gama de resultados que devem ser medidos (Lowrance, 2010).

Finalmente, sublinha a importância do treinamento para desenvolver a perícia na especialidade, promover o oncologista vis-à-vis o cirurgião-geral e capacitá-lo a ocupar o lugar da cirurgia em hospitais gerais, com vistas a referenciar o paciente para centros mais experientes e unidades especializadas com gestão multidisciplinar e crescente subespecialização, assim como a concentrar os tratamentos cirúrgicos complexos nas mãos de cirurgiões especializados (Lowrance, 2010).

Em contraste com os argumentos sobre a relação inversa entre a centralização da estrutura organizacional e o desempenho, os estudos das Ciências da Saúde trazem evidências dignas de nota. Por exemplo, no câncer urológico, um grande volume de cirurgias está associado com melhor controle do câncer e taxas de morbimortalidade mais baixas; na cirurgia do câncer de esôfago, a quantidade de procedimentos é um determinante importante da qualidade do cuidado; no caso do câncer de pâncreas, a concentração do tratamento em grandes unidades aumenta as taxas de incidência de cirurgias, reduz a mortalidade no pós-operatório e aumenta a taxa de sobrevivência; a centralização do cuidado possibilita um significativo aumento da sobrevida do paciente com câncer retal no longo prazo; a centralização da oncologia ginecológica viabiliza o aumento da taxa de preservação da fertilidade das pacientes sob cuidados; o tratamento dos pacientes com câncer do ovário por especialistas em oncologia ginecológica obtém melhores resultados do que o tratamento por cirurgiões e ginecologistas gerais; finalmente, a centralização da cirurgia colorretal resulta no au- 
mento da sobrevida e na queda da mortalidade e do número de novas cirurgias no período pós-operatório (Birgisson \& Smedh, 2009; Kesic, Rodolakis, Denschlag, Schneider, Morice, Amant, \& Reed, 2010; Lemmens, Bosscha, Schelling, Brenninkmeijer, Coebergh, \& Hingh, 2011).

Quanto às limitações da pesquisa sobre centralização, frequência, resultado funcional da cirurgia e controle do câncer nas Ciências da Saúde, alguns autores sustentam que os resultados subestimam os efeitos: do deslocamento do paciente para longe da sua comunidade e da consequente propensão a postergar ou evitar a cirurgia; da orientação multidisciplinar a respeito dos riscos e do acompanhamento sobre a decisão do paciente; da transposição de processos usados em grande escala para unidades menores; das margens existentes para meIhorar o desempenho das unidades menores; de outros fatores sobre o resultado do tratamento; da preferência do paciente de melhor condição econômica pela unidade maior; da variância do desempenho das unidades maiores e dos cirurgiões experientes; e dos recursos superiores ao alcance das unidades maiores (Kesic, Rodolakis, Denschlag, Schneider, Morice, Amant, \& Reed, 2010; Lowrance, 2010; Wouters, Karim-Kos, Cessie, Wijnhoven, Stassen, Steup, Tilanus, \& Tollenaar, 2009).

Uma vez que esses resultados não recorrem a dados longitudinais, são considerados insuficientes para confirmar uma relação causal entre volume de procedimentos complexos e resultado efetivo do tratamento, bem como para prescrever escalas mínimas em bases consistentes, apontando para a necessidade de pesquisas futuras sobre as consequências da centralização para o acesso à atenção, o comportamento dos custos no curto e no longo prazo, a disponibilidade de mão de obra especializada e a prestação dos outros serviços no hospital (Harrison, 2012).

\section{METODOLOGIA}

O objetivo geral desta pesquisa empírica e quantitativa sobre a atividade de diagnóstico de patologia do INCA é avaliar o efeito da centralização dessa atividade sobre o desempenho dos hospitais públicos de tratamento do câncer, abordados como unidades produtivas que são (Schwartzman, 1994).

Cada NHP do INCA adota um foco derivado da especialização do HC a que está associado e engloba, em simultâneo, atividades especializadas, mas diversificadas, de assistência, ensino e pesquisa sobre o câncer, além do apoio administrativo ao conjunto dessas atividades.

A centralização dos NHPs incluiu a fusão dos serviços do $\mathrm{HC}_{1}$ e do $\mathrm{HC}_{3}$ em 1999 e a incorporação do serviço do HC2 em 2002, sob a coordenação da Dipat (Zardo, Fialho, \& Guimarães, 2003).

Estruturas organizacionais reguladas pelo regime da Administração Pública e com objetivos e meios diversificados, os NHPs têm o desempenho condicionado pelos problemas de coordenação e compromisso envolvidos no repasse interno dos recursos do orçamento público anual prefixado do INCA, em face das metas - potencialmente concorrentes por recursos de suas diferentes etapas e linhas de produtos.

O primeiro objetivo específico deste estudo, portanto, é calcular indicadores da evolução comparativa do desempenho dos NHPs prestadores de serviços de diagnóstico de suporte aos HCs do INCA como unidades produtivas, antes e depois da centralização do SAP, em 2002. O segundo objetivo específico é avaliar o efeito global da centralização dos três NHPs sobre a Dipat.

0 que se procura em unidades operacionais tais como o SAP é uma medida global, que relaciona média ponderada de outputs com média ponderada de inputs e em que os pesos representam a importância relativa de cada output e input. A avaliação da estratégia de centralização gerencial do SAP envolve, de início, construir um Indicador Gerencial (IG) para o desempenho conjunto dos NHPs da Dipat, que equivale à média anual dos quocientes entre a soma ponderada dos produtos obtidos e a soma ponderada dos recursos utilizados na produção em cada NHP no período da análise e, a seguir, comparar a evolução desse IG nos subperíodos anterior e posterior à centralização do SAP.

Do ponto de vista da análise não paramétrica, a essa hipótese de pesquisa está associada a seguinte hipótese nula, que será objeto dos testes:

"A centralização do SAP na Dipat-INCA não está diretamente associada à obtenção de ganhos de eficiência no uso de recursos para a realização dos exames de patologia usados no tratamento de pacientes com câncer."

Para examinar os dados levantados, foi usado um modelo Análise Envoltória de Dados (DEA) de análise de eficiência. Esse modelo permite calcular, para o período 1997-2007, os escores-síntese anuais de eficiência técnica relativa dos três NHPS do SAP.

\section{Análise Envoltória de Dados (DEA)}

A seleção de um padrão de comparação entre organizações é tratada na literatura sobre avaliação pela identificação de fronteiras de eficiência. Os modelos de ajuste não paramétrico da fronteira, tais como a DEA, representam o ajuste da fronteira de produção à produção máxima empiricamente observada de qualquer Unidade de Tomada de Decisão (DMU) da população 
estudada. Postulam, nesse sentido, a existência das chamadas ineficiências não alocativas, decorrentes de motivos que escapam ao controle dos gerentes do processo produtivo, cujo comportamento, sem informação completa sobre a produção, seria o de emular o desempenho dos pares.

Aplicados a um conjunto de L organizações que utilizam $\mathrm{N}$ inputs para produzir M outputs, os Modelos DEA possibilitam otimizar os escores-síntese $E_{j}=Y_{j} / X_{j}=\left(A_{1 j} \cdot O_{1 j}+\ldots+A_{M j} \cdot O_{M j}\right) /\left(B_{1 j}\right.$ $\left.\mathrm{I}_{1 \mathrm{j}}+\ldots+\mathrm{B}_{\mathrm{Nj}} \mathrm{I}_{\mathrm{Nj}}\right)$ para cada organização $(\mathrm{j}=1,2, \ldots, \mathrm{L})$, os quais permitem hierarquizar as unidades segundo seu desempenho, pois especificarão um padrão numérico de comparação. Nessa expressão, os Os representam outputs, os Is representam inputs e os As e Bs representam pesos a serem calculados, ou seja, a importância relativa de cada output e input.

Os Modelos DEA-CCR usados para otimizar $\mathrm{E}_{\mathrm{j}}$ são: o Modelo DEA-CCR orientado para insumos, que minimiza o consumo agregado de recursos, mantendo constante a produção; e o Modelo DEA-CCR com orientação para produtos, que maximiza a produção, mantendo constante o consumo agregado de recursos. Os Modelos DEA-BCC, orientados para a redução dos insumos ou orientados para o aumento do produto, apresentam superfícies de fronteira com retornos variáveis de escala (Coelli, Rao, \& Battese, 1998).

No Gráfico 1, por exemplo, a situação relativa de UI, quando o seu desempenho é comparado com o de U1 e U2, permite concluir que, entre as três unidades sob análise, se alguma pertence à fronteira eficiente, é UI, uma vez que utiliza o mesmo montante de recursos XI para produzir $Y_{1}$, ou seja, menos do que $\mathrm{YI}$, enquanto U2 produz o mesmo que UI, mas utiliza um volume $X_{2}$ de recursos maior do que XI. Nesse sentido, a ineficiência técnica de U2 resulta em escore-síntese menor do que o de UI, da mesma forma que a ineficiência de U1 é expressa por escore menor do que o de $U_{3}$.

\section{Gráfico 1. Representação do escore-síntese de eficiência técnica}

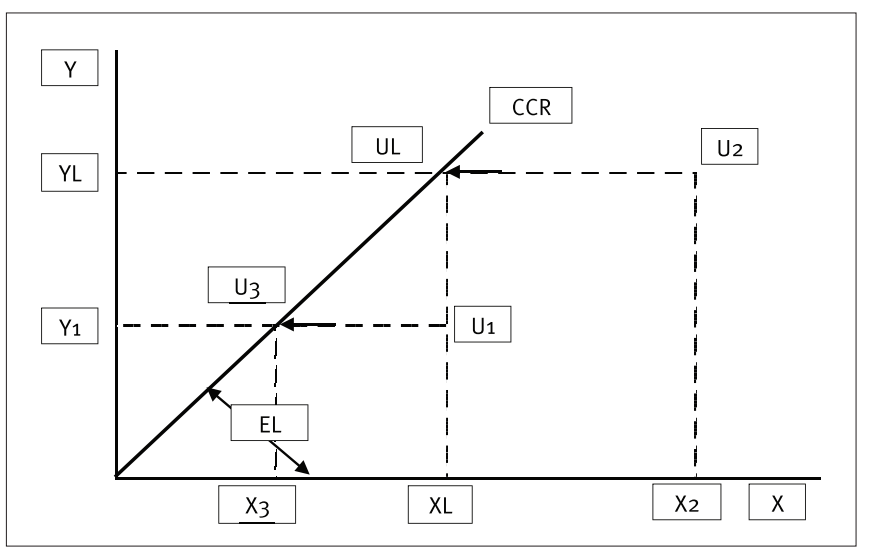

Convenciona-se, a propósito, que $E_{j}=Y_{j} / X_{j}=1$ para todas as organizações j situadas na fronteira, ou seja, a situação de eficiência plena está caracterizada de modo equivalente para todas elas como unidades $100 \%$ eficientes. Assim, a eficiência relativa $E_{1}$ de uma unidade $U_{1}$ cujo ponto representativo não pertence à fronteira eficiente pode ser medida como $E_{1}=Y_{1} / X_{1}<1$ e $E_{1}$ é um escore-síntese para seu desempenho - veja o modelo no Gráfico 2.

\section{Gráfico 2. Representação da fronteira eficiente}

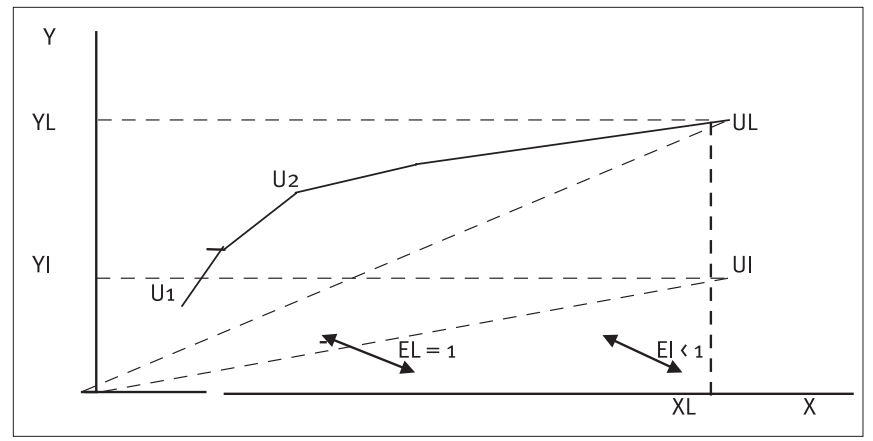

Além disso, por transformação dos quocientes entre os escores $E_{1} / E_{j}$, pode ser computada uma estimativa dos ganhos - medidos em termos do aumento esperado da produção ou da economia de insumos - que podem resultar da transferência dos recursos e da responsabilidade pela produção de uma unidade ineficiente para uma unidade eficiente, ou seja, dos novos planos de operação pró-eficiência prescritos.

Neste trabalho, foi usado o programa DEAP versão 2.1 - Data Envelopment Analysis Computer Program - para o cálculo da fronteira de produção pela técnica DEA (Coelli, 1996).

As vantagens oferecidas pelas técnicas não paramétricas de análise de dados - tais como o Modelo DEA - para a condução dos estudos de avaliação comparativa são significativas, mas requerem cautela quanto à validação dos resultados (Ozcan, 2008). Por um lado, possibilitam a análise de variáveis de recursos utilizados e resultados obtidos relativas a amostras das organizações avaliadas cujas distribuições nas populações de origem muitas vezes são desconhecidas, e de variáveis quantificadas em diferentes unidades de medida, uma vez que a eventual inexistência de mercados completos para os bens e serviços envolvidos implica o desconhecimento de preços e valores (Coelli, 1996). No entanto, segundo o procedimento consagrado na literatura de aplicações do Modelo DEA à análise de eficiência, a robustez genérica dos resultados não paramétricos pode ajudar a suavizar o problema da sensibilidade à variação amostral, na medida em que 
é assegurada pela aplicação dos testes estatísticos apropriados (Siegel, 1956).

\section{Testes de aferição dos resultados}

Uma vez que as amostras disponíveis provêm de populações com distribuição de probabilidades desconhecida, os testes não paramétricos de Mann-Whitney e Friedman foram utilizados para analisar os escores-síntese calculados com o Modelo DEA e lidar com o problema de indeterminação do erro de cálculo inerente ao método determinístico escolhido.

$O$ teste $U$ de Mann-Whitney é usado para testar se duas amostras independentes foram retiradas de populações com médias iguais. A hipótese nula a ser testada é que não há diferença entre (as médias dos) os grupos estudados. A distribuição U pode ser aproximada por uma normal reduzida $Z$ de modo que, se $\left.z_{\text {calc }}\right\rangle|c|$ ao nível de significância $a=0,01$, rejeita-se a $1 \%$ a hipótese nula de que não há diferença entre os dois grupos (Martins, 2002).

$\mathrm{O}$ teste $\mathrm{F}$ de Friedman é usado para testar a existência de diferença entre $K>2$ amostras não necessariamente independentes. A hipótese nula a ser testada é de que não há diferença entre as $\mathrm{K}$ amostras. Usando a aproximação do qui-quadrado, se $\mathrm{F}_{\text {calc }}$ ' $\mathrm{x}^{2}$ com $\mathrm{K}-1$ graus de liberdade e $\mathrm{a}=0,05$, rejeita-se a $5 \%$ a hipótese nula de que as amostras são estatisticamente indistintas, mesmo que extraídas da mesma população (Siegel, 1956).

\section{Universo, amostra e coleta de dados}

O universo da pesquisa é formado pelos quatro NHPs da Dipat. Uma vez que apenas o $\mathrm{HC}_{1}$, o $\mathrm{HC}_{2}$ e o $\mathrm{HC}_{3}$ têm atividade cirúrgica que requer um serviço de patologia, a avaliação da eficácia da estrutura organizacional do SAP concentrou-se na análise de eficiência desses NHPs.

Os dados básicos retroativos a 1997 foram coletados em três fontes: nos bancos de dados do Sistema de Anatomia Patológica do INCA (Siapi); nas escalas de trabalho e na folha de pessoal do INCA; e nos relatórios da equipe de informática do SAP (Santiago, 2009).

0 resultado do diagnóstico do paciente, o laudo, é o principal produto do SAP (Vassallo, 2003). Consiste da identificação e classificação dos processos patológicos por meio do método morfológico pelo médico patologista, permitindo ao corpo clínico adotar terapêuticas adequadas a cada caso.

O "recebimento do material no NHP" é o ponto de partida da cadeia de valor que integra as atividades das unidades produtivas do SAP. Com a centralização das atividades do SAP a partir de 2002, o material originário dos três NHPs passou a ser processado na Dipat. Esse material provém basicamente de serviços externos (exames externos), ambulatoriais (biópsias) e cirúrgicos (peças), é registrado com um número único no Siapi e um médico patologista é designado responsável.

Além do médico, essa atividade requer profissionais que deem suporte administrativo e sejam responsáveis pela produção de lâminas com o material a ser examinado. Este estudo destaca a produção das lâminas de hematoxilina e eosina (HE), consideradas a base do diagnóstico de patologia, além de abranger as eventuais solicitações de "exames complementares" para auxiliar o diagnóstico (imuno-histoquímica ou coloração especial, por exemplo).

Se não há necessidade de nenhum exame complementar, o exame é liberado, e ocorre a sua assinatura digital pelo patologista responsável.

O "laudo final" é resultado da assinatura de todos os exames histopatológicos, que podem ter mais de um diagnóstico, conforme o material analisado. Esse diagnóstico é codificado segundo a Classificação Internacional de Doenças para Oncologia (CID-0). Com a assinatura do caso completo, as lâminas são finalmente arquivadas, tendo importância para o diagnóstico de exames futuros do paciente e para a pesquisa.

Por fim, o "tempo médio de emissão do laudo" conta o número de dias decorridos entre a data do registro e a assinatura do caso, um indicador usualmente utilizado para medir a qualidade do serviço.

Quanto aos recursos variáveis que constituem restrições no curto prazo para a "emissão do laudo" pelo SAP, incluem, portanto, o número de médicos patologistas e de profissionais não médicos, o de lâminas arquivadas e o de "pacientes atendidos nos NHPs", uma vez que a decisão sobre a necessidade do exame do paciente ocorre fora do SAP.

Quatro inputs - a saber, Número de Pacientes/ano do SAP, Número de Médicos Patologistas com Assinatura de Laudo/ano, Número de Horas de Profissional Não Médico/ano e Número de Blocos Arquivados/ano - e quatro outputs - Número de Diagnósticos Histopatológicos Codificados/ano, Número de Exames Histopatológicos/ano, Número de Laudos Histopatológicos/ano e Número Médio de Dias para Emissão de Laudo por Patologista/ ano foram, então, considerados para a especificação do Modelo DEA usado na análise de eficiência dos NHPs e do SAP/INCA.

O período coberto pela série de dados anuais disponíveis - 11 anos - resultou em um número de unidades de observação por variável de interesse (4,12 unidades) compatível com a recomendação da literatura sobre o poder de discriminação do Modelo DEA, de modo que foi possível calcular uma fronteira única para o conjunto dos NHPs anuais no período 1997-2007. (Coelli, Rao, \& Battese, 1998). 


\section{RESULTADOS}

Os resultados expostos nesta seção estão distribuídos em duas subseções. Primeiro, os NHPs-ano relativamente “eficientes” e relativamente "ineficientes" são exibidos. A subseção seguinte analisa os resultados dos testes estatísticos da diferença entre os escores.

\section{Classificação dos NHPs segundo sua eficiência}

O problema de cálculo do Modelo DEA-CCR-O foi resolvido para a obtenção dos escores-síntese que distinguem os 33 NHPs-ano entre eficientes e não eficientes - veja a Tabela 1. Esse modelo foi escolhido para a análise de eficiência dos NHPs, uma vez que: a. a escolha do gerente é dirigida a maximizar os outputs, pois o orçamento do SAP é prefixado (os inputs são os disponíveis no início do ano);

b. o período coberto pela análise é longo o suficiente para contemplar as situações de equilíbrio de longo prazo implícitas na hipótese de retornos constantes de escala do modelo de livre concorrência; e

c. o Modelo DEA-CCR distinguiu melhor os grupos de NHPs do que o Modelo DEA-BCC.

A Tabela 1 permite monitorar cada NHP-ano individualmente no decorrer do período da análise, avaliando a performance de cada um, e comparar seu desempenho em relação ao do conjunto dos NHPs-ano, o que também pode ser visualizado no Gráfico 3.

\section{TABELA 1. Escores-síntese de eficiência dos NHPs I (em \%)}

\begin{tabular}{|c|c|c|c|c|c|c|}
\hline \multicolumn{7}{|c|}{ Modelo DEA-CCR-O com especificação de oito variáveis } \\
\hline $\mathrm{NHP}_{1}$ & 100,00 & 100,00 & 100,00 & 100,00 & 100,00 & 100,00 \\
\hline $\mathrm{NHP}_{2}$ & 100,00 & 100,00 & 100,00 & 80,70 & 69,00 & 83,40 \\
\hline $\mathrm{NHP}_{3}$ & 81,40 & 100,00 & 100,00 & 100,00 & 95,90 & 98,20 \\
\hline NHPs & 2003 & 2004 & 2005 & 2006 & 2007 & \\
\hline NHP1 & 99,30 & 100,00 & 100,00 & 99,60 & 98,80 & \\
\hline $\mathrm{NHP} 2$ & 83,80 & 91,70 & 87,80 & 90,70 & 88,20 & \\
\hline
\end{tabular}

\section{Gráfico 3. Evolução anual do escore-síntese médio: 1997-2007}

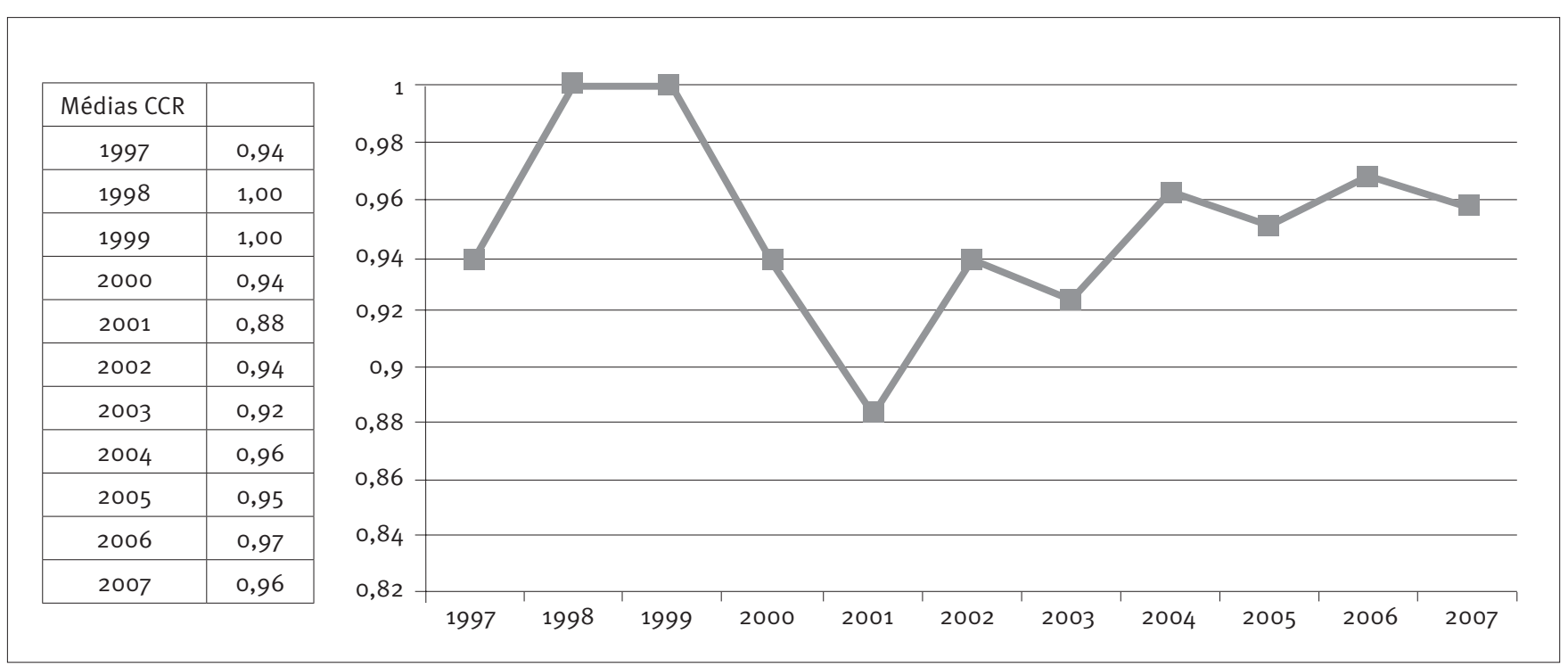


O Modelo DEA-CCR-O revelou poder explicativo com respeito à heterogeneidade do desempenho das unidades produtivas. Como pode ser observado na Tabela 1, 17 das 33 DMUs examinadas estão fora da fronteira de eficiência.

Em geral, as unidades ineficientes ficaram próximas da fronteira de eficiência, o que pode significar efeito aprendizado cruzado entre os gerentes ou rigidez das condições de processo (coeficientes técnicos fixos, por exemplo), também sugerida pelo fato de que a melhor distinção entre os grupos de unidades eficientes e ineficientes foi obtida a partir dos escores-síntese computados com o Modelo DEA-CCR.

A evolução dos escores-síntese médios de cada ano, que pode ser observada no Gráfico 3, indica, em resumo, perda de eficiência entre 1999 e 2001 e ganhos a partir de 2002.

Diante da hipótese de pesquisa deste estudo, a análise comparativa da média anual dos escores calculados é de especial interesse, uma vez que: a) pode confirmar a hipótese de associação entre a mudança da estrutura organizacional do SAP e a melhor performance dos NHPs no período pós-centralização gerencial se houve variação positiva do escore médio dos NHPs a partir de 2002; e b) pode sugerir perda de eficiência se, ao contrário, acusar variação negativa. De fato, de um lado, houve queda do valor do escore médio anual de eficiência calculado no período 1998-2001 (de 100\% em1998 até 88,3\% em 2001) e, de outro, houve aumento no período 2002-2007 (de 93,9\% em 2002 até 95,7\% em 2007) - veja a Tabela 1. De imediato, portanto, esses resultados podem ser interpretados como confirmação da hipótese de pesquisa.

\section{Resultados do teste de precisão dos resultados}

Diante da variabilidade relativamente baixa dos escores-síntese que distinguem os NHPs-ano, uma questão adicional a verificar é se os NHPs apresentaram variação significativa do coeficiente médio de eficiência técnica relativa de um ano para outro.

Comparados os escores de eficiência dos grupos de 16 NHPs-ano "eficientes" e 17 "ineficientes" por meio do teste de Mann-Whitney, obteve-se Z $<-2,3$, rejeitando a hipótese nula de que as duas amostras de NHPs fazem parte da mesma população, ou seja, existe diferença estatisticamente significativa entre os escores dos NHPs distinguidos nos grupos ( $p<0,01)$.

Quanto à avaliação da diferença dos escores dos NHPs de diversos anos pelo teste de Friedman, uma vez que a estatística F, calculada para 11 amostras de três elementos, alcançou o valor 446,3, maior que o valor teórico aproximado pela distribuição de qui-quadrado com 10 graus de liberdade e $95 \%$ de confiança, rejeita-se a hipótese nula, ou seja, pode-se afirmar que existe diferença estatisticamente significativa entre os escores médios de eficiência técnica de diversos anos.

\section{CONCLUSÕES}

O uso do Modelo DEA-CCR-O neste artigo mostrou-se frutífero para extrair contribuições da avaliação da eficácia da mudança de estrutura organizacional do SAP em duas direções. Ao avaliar essa reestruturação organizacional à luz do princípio da análise de eficiência, a modelagem DEA aportou conhecimento sobre a Administração em geral, assim como trouxe uma contribuição para a melhoria operacional da atividade de anatomia patológica dos hospitais públicos especializados em oncologia.

$\mathrm{Na}$ literatura internacional, a propósito, desde há muito a maioria das aplicações da DEA no setor saúde, a despeito de sua importância quantitativa e qualitativa, continua concentrada na avaliação do desempenho operacional de unidades prestadoras de serviços comparadas no mesmo momento (Blank \& Valdmanis, 2008, p. 238; Fare, Grosskopf, Lundstrom, \& Rose, 2008; Hollingsworth, Dawson, \& Maniadakis, 1999).

Tomando a perspectiva do caminho temporal da eficiência ao longo do período escolhido, no entanto, a abordagem DEA também foi empregada neste trabalho para avaliar uma mudança organizacional à luz do critério de eficácia de Costa (2000), de modo que, na presente aplicação, o principio de avaliação foi o mesmo, mas, considerada a evolução temporal, o escopo foi muito mais organizacional do que operacional. Nesse contexto, o Modelo DEA pode ser considerado um caminho capaz de dar sentido empírico (ou "prático") à questão da avaliação da influência da centralização sobre a eficiência organizacional, desde há muito presente na literatura (Etzioni, 1967, p. 48).

Embora a utilização da DEA para avaliar organizações sob uma perspectiva mais abrangente tenha registro na literatura internacional (por exemplo, Lee, Bott, Gajewski, \& Taunton, 2009), o presente trabalho oferece contribuição pioneira, no caso brasileiro, acerca da avaliação quantitativa do efeito da centralização da tomada de decisão sobre o desempenho da unidade produtiva. Diversos resultados aqui obtidos apontam nessa direção.

Quanto ao conhecimento sobre a Administração em geral, a literatura de Administração e Economia sobre a formação das vantagens competitivas da firma serve de suporte à noção básica de que a centralização é um obstáculo ao desenvolvimento da orientação empreendedora na base operacional e no staff e compromete o posicionamento competitivo, enquanto a literatura estrangeira recente de Ciências da Saúde sobre a re- 
lação entre o volume de atividade e o resultado da adoção de procedimentos cirúrgicos nos hospitais de tratamento do câncer acumula evidências de ganhos de efetividade clínica associados à centralização do atendimento nos hospitais de grande porte.

Como resultado do estudo de caso sobre os NHPs do INCA, no entanto, foram obtidas evidências em suporte à validação da hipótese explicativa do Modelo DEA sobre a escoIha da estrutura organizacional nas organizações públicas multipropósito, em circunstâncias nas quais o uso de indicadores convencionais costuma colocar o avaliador em posição inconclusiva, como é o caso em unidades de produção multipropósito. Identificados pelo estudo, os ganhos de eficiência obtidos com a implementação da estratégia de centralização dos NHPs na DIPAT no período da análise permitiram confirmar a hipótese alternativa de racionalidade econômica implícita no Modelo DEA-O: os gerentes espelham-se nos programas operacionais dos seus "pares" da fronteira eficiente em busca de melhorias de desempenho.

Quanto à contribuição gerencial, ao identificar ganhos de performance nos NHPs, a análise de eficiência desenvolvida ofereceu evidências de que a possibilidade de a centralização comprometer a atitude de competição agressiva, inovadora, proativa, de tomada de risco e de autonomia e da capacidade adaptativa não desqualifica a eficácia da estrutura de tipo Dipat para dar suporte laboratorial aos hospitais do INCA.

Os resultados obtidos no nível de agregação examinado com a aplicação do Modelo DEA deste estudo apontam, para o conjunto dos NHPs, um aumento médio de eficiência de $8,3 \%$ no período 2002-2007 de advento da centralização, ou seja, indicam que, após seis anos dessa reestruturação, uma combinação equivalente à das quantidades utilizadas de pacientes encaminhados, médicos, profissionais não médicos e material coletado arquivado na patologia do INCA em 2001 seria suficiente para assegurar uma quantidade combinada de exames complementares, laudos finais, diagnósticos registrados e redução de tempo de emissão de laudos $8,3 \%$ maior. Significam, alternativamente, que a mesma combinação de resultados da patologia do INCA antes da centralização foi possível com uma redução da ordem de $8,3 \%$ dos recursos requeridos na nova estrutura organizacional da Dipat - e dos dispêndios anuais do SUS.

Nesse sentido, a análise de eficiência oferece um marco conceitual elucidativo e uma ferramenta informativa para o conhecimento e a tomada de decisão sobre a mudança de estrutura nas organizações públicas multipropósito de saúde.

Finalmente, apesar de a circunscrição da análise de eficiência a um específico laboratório de patologia poder constituir uma limitação à generalização dos resultados obtidos acer- ca do efeito da centralização dessa unidade operacional sobre o desempenho de quaisquer hospitais de oncologia, é preciso enfatizar, igualmente, que:

- persiste a falta de padronização dos conceitos de input e output usados no estágio atual da pesquisa sobre o hospital como unidade produtiva (Sherman \& Zhu, 2006);

- é importante operacionalizar essas variáveis de input e output dos hospitais utilizando as medidas disponíveis nas bases de dados existentes (Ozcan, 2008);

- a obtenção dos dados necessários à avaliação demandou esforço de inventário sem precedentes na Dipat, mesmo que tenha contribuído para o autoconhecimento na organização e seja repetidamente reconhecida como crucial (Blank \& Valdmanis, 2008, p. 238);

- algumas limitações da análise poderão ser contornadas com o conhecimento das características e do contexto de cada NHP; e

- a aferição dos resultados depende, em particular, de mais conhecimento acerca das implicações do aprendizado para a existência de relacionamento entre as técnicas utilizadas nos NHPs.

\section{REFERÊNCIAS}

Alimardani, M, Ghahremani, M, \& Abulghesemi, M. (2009). Examination of relation between organizational structure and organizational entrepreneurship. A New Advancement into Educational Management, 3, 131-144.

Birgisson, H, \& Smedh, K. (2009). Effects of centralization of colorectal surgery on the outcome of patients with distal sigmoid colonic cancer. Digestive Surgery, 26(2), 169-175.

Blank, J. L. T, \& Valdmanis, V. G. (2008). Efficiency in hospital industry: summary and conclusions. In J. L. T. Blank, \& V. G. Valdmanis (Eds.). Evaluating hospital policy and performance: contributions from hospital policy and productivity research (Vol. 18, pp. 231-240). Bingley, UK: Emerald Group Publishing.

Caruana, A, Ewing, M. T, \& Ramaseshan, B. (2002). Effects of some environmental challenges and centralization on the entrepreneurial orientation and performance of public sector entities. The Service Industries Journal, 22(2), 43-58.

Coelli, T. (1996). A guide to DEAP version 2.1: a data envelopment analysis (computer) program [Working Paper No 8]. CEPA, University of New England, Australia.

Coelli, T, Rao, D. S. P, \& Battese, G. E. (1998). An introduction to efficiency and productivity analysis. Boston: Kluwer.

Costa, C. B. e. (200o). Entrevista. Boletim da SOBRAPO. Informe n. 20. Rio de Janeiro: SOBRAPO.

Costa-I-Font, J. (2012). Myths of health care decentralization. Journal of Health Services Research \& Policy, 17(4), 252-253. 
Etzioni, A. (1967). Organizações modernas (6a ed.). São Paulo: Livraria Pioneira Editora.

Fare, R, Grosskopf, S, Lundstrom, M, \& Roos, P. (2008). Evaluating health care efficiency. In J. L. T. Blank, \& V. G. Valdmanis (Eds.). Evaluating hospital policy and performance: contributions from hospital policy and productivity research (Vol. 18, pp. 209-228). Bingley, UK: Emerald Group Publishing.

Faria, P. A. S. (2007). Inovação na organização dos serviços de anatomia patológica dos hospitais federais do Rio de Janeiro: a construção de um modelo para a rede de atenção oncológica. Dissertação de mestrado, Escola Nacional de Saúde Pública, Rio de Janeiro.

Harrison, A. (2012). Assessing the relationship between volume and outcome in hospital services: implications for service centralization. Health Services Management Research, 25(1), 1-6.

Hollingsworth, B, Dawson, P. J, \& Maniadakis, N. (1999). Efficiency measurement of health care: a review of non-parametric methods and applications. Health Care Management Science, 2(3), 161-172.

Jogaratnam, G, \& Tse, E. C.-Y. (2006). Entrepreneurial orientation and the structuring of organizations: performance evidence from the Asian hotel industry. International Journal of Contemporary Hospitality Management, 18(6), 454-468.

Johnson, S, \& Ven, A. H. Van de. (2002). A framework for entrepreneurial strategy. In M. A. Hitt, R. D. Ireland, S. M. Camp, \& D. L. Sexton (Eds.). Strategic entrepreneurship: creating a new mindset (pp. 66-85). Oxford, UK: Blackwell Publishers.

Jorge, M. J. (2001). Mensuração de performance e modelo de gestão segundo resultados em organizações de C\&T do campo da saúde: uma avaliação da experiência da FIOCRUZ. Tese de doutorado, COPPE/UFRJ, Rio de Janeiro.

Katsikea, E, Theodosiou, M, Perdikis, N, \& Kehagias, J. (2011). The effects of organizational structure and job characteristics on export sales managers job satisfaction and organizational commitment. Journal of World Business, 46(2), 221-233.

Kesic, V, Rodolakis, A, Denschlag, D, Schneider, A, Morice, P, Amant, F, \& Reed, N. (2010). Fertility preserving management in gynecologic cancer patients: the need for centralization. International Journal of Gynecological Cancer, 20(9), 1613-1619.

Lee, R. H, Bott, M. J, Gajewski, B, \& Taunton, R. L. (2009). Modeling efficiency at the process level: an examination of the care planning process in nursing homes. Health Services Research, 44(1), 15-32.

Lemmens, V. E, Bosscha, K, Schelling, G. van der, Brenninkmeijer, S, Coebergh, J. W. W, \& Hingh, I. H. J. T. (2011). Improving outcome for patients with pancreatic cancer through centralization. British Journal of Surgery, 98(10), 1455-1462.

Lowrance, W. T. (2010). Surgical margins following urological cancer surgery and clinical outcomes - does the United States need regional centralization of cancer care? The Journal of Urology, 183(6), 2126-2127.

Luft, H. S, Bunker, J. P, \& Enthoven, A. C. (1979). Should operations be regionalized? The empirical relation between surgical volume and mortality. The New England Journal of Medicine, 301(25), 1364-1369.
Lumpkin, G. T, Cogliser, C. C, \& Schneider D, R. (2010). Understanding and measuring autonomy: an entrepreneurial orientation perspective. Entrepreneurship Theory and Practice, 33(1), 47-69.

Martins, G. A. (2002). Estatística geral e aplicada (2a ed.). São Paulo: Atlas.

Meirovich, G, Brender-Ilan, Y, \& Meirovich, A. (2007). Quality of hospital service: the impact of formalization and decentralization. International Journal of Health Care Quality Assurance, 20(3), 240-252.

Mintzberg, H. (1995). Criando organizações eficazes. São Paulo: Atlas.

Morton, A. N, \& Hu, Q. (2008). Implications of the fit between organizational structure and ERP: a structural contingency theory perspective. International Journal of Information Management, 28(5), 391-402.

Ozcan, Y. A. (2008). Health care benchmarking and performance evaluation: an assessment using data envelopment analysis (DEA). Virginia: Springer.

Puccini, P. T. (2008). As unidades de assistência médica ambulatorial (AMA) do Município de São Paulo, Brasil: condições de funcionamento e repercussões sobre a atenção básica no Sistema Único de Saúde, 2006. Cadernos de Saúde Pública, 24(12), 2755-2766.

Robinson, R. B. (2007). Creating a 21st century entrepreneurial organization. Academy of Entrepreneurship Journal, 8(1), 321-332.

Santiago, R. P. (2009). Análise de eficiência de organizações de saúde: o caso da anatomia patológica do INCA. Monografia de conclusão do curso de Ciências Econômicas, Faculdade de Economia, Universidade Federal Fluminense, Niterói.

Schwartzman, J. (1994, maio). Um sistema de indicadores para as universidades brasileiras [Mimeo]. NUPES/USP, São Paulo.

Shepherd, D, Covin, G. F, \& Kuratko, F. D. (2008). Project failure from corporate entrepreneurship: managing the grief process. Journal of Business Venturing, 24(6), 588-600.

Sherman, H. D, \& Zhu, J. (2006). Service productivity management: improving service performance using Data Envelopment Analysis (DEA). Boston, MA: Springer.

Siegel, S. (1956). Nonparametric statistics. New York: McGraw-Hill.

Stitzenberg, K. B, Wong, Y. N, Nielsen, M. E, Egleston, B. L, \& Uzzo, R. G. (2012). Trends in radical prostatectomy: centralization, robotics, and access to urologic cancer care. Cancer, 118(1), 54-62.

Vakkuri, J. (2003). Research techniques and their use in managing non-profit organizations: an illustration of DEA analysis in NPO environments. Financial Accountability and Management, 19(3), 243-263.

Vassallo, J. (2003). Controle de qualidade e acreditação no laboratório de anatomia patologia. São Paulo: Sociedade Brasileira de Patologia.

Wouters, M. W. J. M, Karim-Kos, H. E, Cessie, S. le, Wijnhoven, B. P. L, Stassen, L. P. S, Steup, W. H, Tilanus, H. W, \& Tollenaar, R. A. E. M.. (2009). Centralization of esophageal cancer surgery: does it improve clinical outcome. Surgical Oncology, 16(7), 1789-1798.

Zardo, L, Fialho, F, \& Guimarães, D. S. (2003). A história da patologia no INCA. Revista Brasileira de Cancerologia, 49(1), 59-61. 